\title{
COUPLED PIEZORESISTIVE GRAPHENE NANO-RESONATORS
}

\author{
Madhav Kumar and Harish Bhaskaran \\ Department of Materials, University of Oxford, United Kingdom
}

Bhaskar Choubey

Chair of analogue circuits and image sensors, Siegen University, Germany

\begin{abstract}
Single atom thick graphene nanomechanical resonators can have very low mass yet high stiffness leading to the potential of very high sensitivity frequency based mass and force sensors. This sensitivity can be further enhanced by coupling two resonators using the Eigenvalues of the response. However, high Q graphene resonators are difficult to manufacture and measure. In this paper, we report the first ever coupled graphene nanoresonator system utilizing the inherent piezoresistivity of graphene. The structure offers mass sensing abilities of zeptogram level using the eigenvectors and quality factor of 1000. It also showcases the potential of making very large coupled arrays to measure more than one substance using a single sensor.
\end{abstract}

\section{INTRODUCTION}

Nano-resonators can provide very high sensitivity for mass sensing leading to the ability of very low mass being detected often in the ranges of biological samples [1-2]. A two dimensional material like graphene can provide enhanced sensitivity and resolution for small mass changes. A number of recent papers have reported such structures [3-7]. However, as the sizes of the nanoresonators have reduced, the same has not been the case of contact pads. This means that significant fabrication area is wasted in the electrical connections to these resonators. Furthermore, despite the small size of the resonators, it is generally not preferred to have more than one moving element on a substrate due to effects of coupling, wherein movement of one resonator affects the performance of another due to parasitic coupling.

However, if one utilizes the effects of coupling rather than avoiding it, one may design mechanical multiplexing in arrays of nano-resonators by utilizing their collective behavior. It is feasible to design multisensing resonant devices by functionalizing different resonators with different sensing abilities [9-13]. Graphene structures are notoriously difficult to fabricate and no suitable transduction mechanism has been reported to utilize coupling in these structures. In this paper, we showcase the potential of graphene coupled resonators by designing, fabricating and testing two-coupled resonators on the same substrate. We utilize the piezoresistivity of graphene as the transduction mechanism [6]. By using a simple inputoutput circuit, we measure the piezoresistivity of a coupled array and record both its resonance frequencies showing the collective behaviour, while reducing the electrical contacts requirement.

The next section presents a theoretical analysis of coupled resonating structures along with a description of the design of the two-coupled structures made of Graphene. Its fabrication using a standard lithography process is explained next. Measurement assembly is then introduced. The test results from the two-coupled resonators are presented using the frequency response of the device. The final section of the paper provides the conclusion.

\section{COUPLED RESONATORS}

A lumped-parameter model of any resonating structure can be described as [1]

$$
m \ddot{x}+B \dot{x}+k x=F
$$

where $x, m, B, k$ and $F$ are its displacement, mass, damping, stiffness and the force applied respectively. The natural undamped resonance frequency of this system would be $\sqrt{k / m}$. Changes in mass or stiffness of the device can be measured by recording its resonant frequency shift. Furthermore, one can record smaller changes in masses, if the mass of the device itself is made smaller.

The sensitivity of the nanoresonator device can be further increased by utilizing two coupled resonators and the eigen-response of a coupled system. A simple coupled system is the one with linear coupling in stiffness [10]

$$
\begin{aligned}
& m_{1} \ddot{x_{1}}+B_{1} \dot{x_{1}}+k_{1} x_{1}+k_{c}\left(x_{1}-x_{2}\right)=F_{1} \\
& m_{2} \ddot{x_{2}}+B_{2} \dot{x_{2}}+k_{2} x_{2}+k_{c}\left(x_{2}-x_{1}\right)=F_{2}
\end{aligned}
$$

Such a system will have two resonance frequencies given by the eigenvectors of the matrix system equation. In addition, at each resonance frequency, the amplitude ratios of the responses of the two resonators are related to the eigenvector of the system. For example, for identical resonators, the response at the first resonance frequency of both devices will be identical in amplitude and phase. However, at the second resonance frequency, they will be out of phase, though with the same amplitude. More importantly, however, the eigenvectors are known to be far more sensitive to small perturbations than the eigenvalues. This means that the eigenvectors can provide higher sensitivity that resonance frequency alone. Nevertheless, the linearity of the eigenvectors is limited [12].

While the aforementioned description of sensory parameters, have included sensing a single substance, one can in effect functionalize each resonator differently. Furthermore, one can record both resonance frequencies from the response of one resonator. Any change to a resonator will lead to change in these resonance frequencies. If the devices are identical, it is impossible to differentiate the origin of these changes. However, nanofabrication rarely, if ever, leads to identical devices [10-12]. This means that the inherent variability of the 
devices will enable one to uniquely determine which resonator has changed while measuring from any one of the resonators, thereby providing inherent mechanical multiplexing.

\section{DESIGN}

Such a multiplexed structure using two resonators built in graphene for very high sensitivity are presented in this paper. Due to its novel nature, standard fabrication processes have not been developed for graphene. Furthermore, a number of different transduction modalities have been suggested. Piezoresitivity of Graphene was selected for our resonators, as it provides for a very simple electrical readout. Furthermore, it has been recently shown to provide high quality resonating structures at room temperature [6].

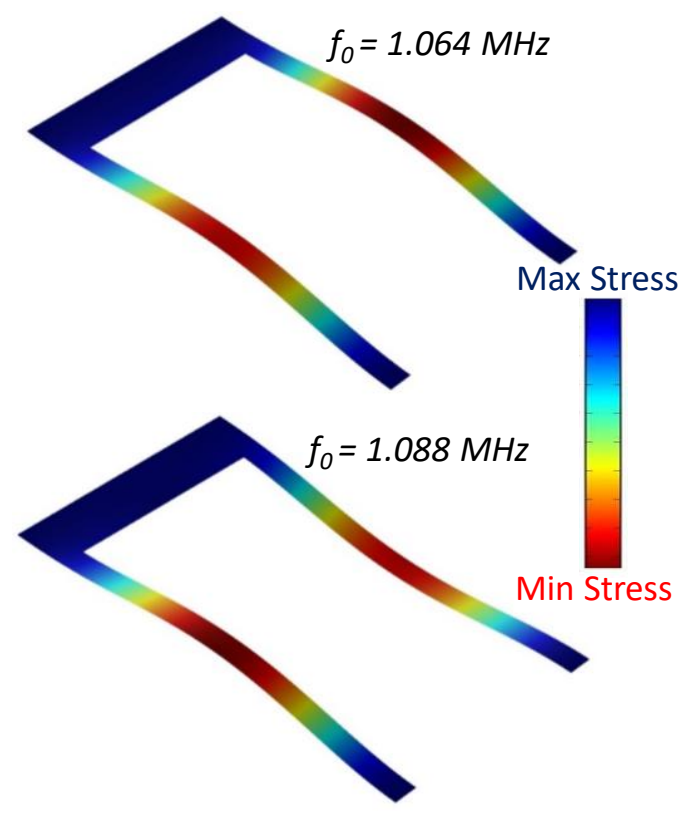

Figure 1: Finite element simulations with coupled resonators made of graphene showing the modal behavior. Two modes are present, one with both arms resonating in-phase and another with resonating outof-phase Color scaler bar shows different parts of the resonator with varying stress distribution.

Clamped-clamped beam resonators have long been studied and provide a simple, yet very useful structure for a range of resonating devices. Two such clamped-clamped beam devices were coupled by using a coupling beam at one end of clamping. Finite element simulations were first carried out to understand their behavior. Figure 1 presents the results of this simulation showing the two resonance modes, wherein the two resonators are either moving inphase or out-of-phase with one another. This therefore, makes the present devices a three-sided clamped structure, providing the abilities of three different readout combinations to measure the resistance between any two arms.

\section{FABRICATION}

Graphene used to build the resonators has been grown using chemical vapour deposition (CVD) process, which has now become a routine process to grow graphene. This CVD grown graphene was transferred on a $300 \mathrm{~nm} \mathrm{SiO}_{2}-$ on-Si wafer for better optical contrast. The three sided clamped-clamped resonating structure was patterned using standard electron beam lithography. The final graphene structure was shaped using reactive ion etching.

Gold electrodes were then deposited for electrical contacts. This was undertaken using yet another e-beam lithography process by patterning electrical contact pads followed by thermal evaporation of $80 \mathrm{~nm}$ gold $(\mathrm{Au})$ on 10 $\mathrm{nm} \mathrm{Cr}$ adhesive layer. Finally, graphene devices were released and hence suspended using a wet etching process. Selective etching of the $\mathrm{SiO}_{2}$ (underneath graphene) is performed using a buffered oxide etchant (BOE). Only partially etching of $\mathrm{SiO}_{2}$ was carried out leaving some $\mathrm{SiO}_{2}$ underneath. However, one can also completely etch $\mathrm{SiO}_{2}$ film. The wet etching process can collapse the graphene structure due to its own surface tension with medium change from liquid to ambient condition. Therefore, after etching the underlying $\mathrm{SiO}_{2}$ layer, the device was dried using a critical point drying mechanism. Herein, we took the liquid medium to a supercritical liquid and then brought it carefully to an ambient condition, thereby avoiding sudden phase change from liquid to gas $[6,14]$.

Figure 2 shows an SEM image of the fabricated structure. Each resonator-arm is $1.75 \mu \mathrm{m}$ long, $150 \mathrm{~nm}$ wide and coupled through a beam with 'd' 180 - 220nm long and $800-900 \mathrm{~nm}$ wide. The two arms were designed to be identical. However, the SEM image shows that they have not been fabricated identically. This is expected expected due to curling of the edges of freestanding graphene structures and can be utilised for mechanical multiplexing.

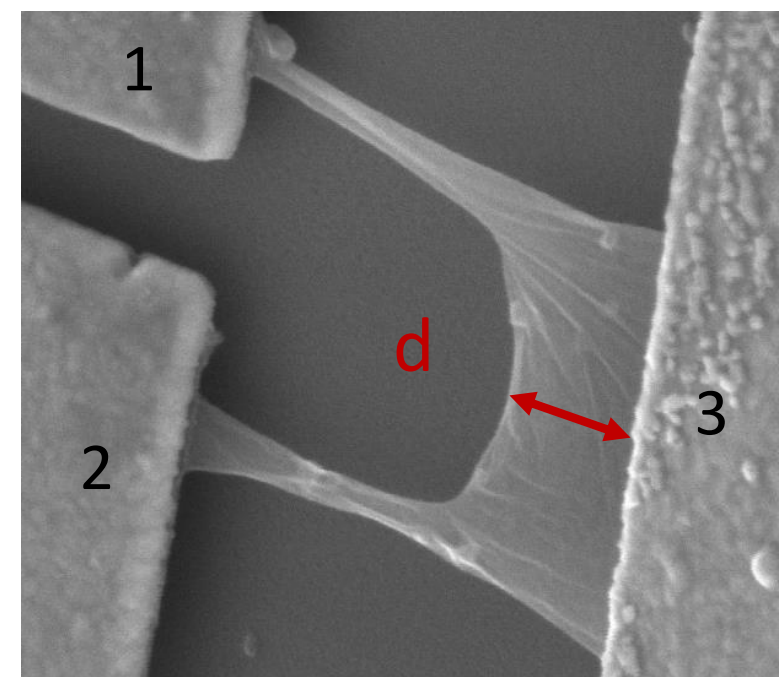

Figure 2: SEM photograph of the coupled graphene resonators

The dimension ' $d$ ' of the coupling beam can be designed with different dimensions to provide different 
amount of coupling between the two resonating beams. Changing the coupling also leads to different separation between the resonance frequencies of the structure, as shown by the finite element simulations in Figure 3. As one varies ' $d$ ', one can see that the two modes come closer on decreasing ' $d$ ' and vice versa. Finite element simulation presented in Figure 1 show the two expected principal modes in-phase $f_{0}=1.064 \mathrm{MHz}$ and out-of-phase $f_{1}=$ $1.088 \mathrm{MHz}$. These two modes are close to each other as expected, when ' $d$ ' is relatively small.

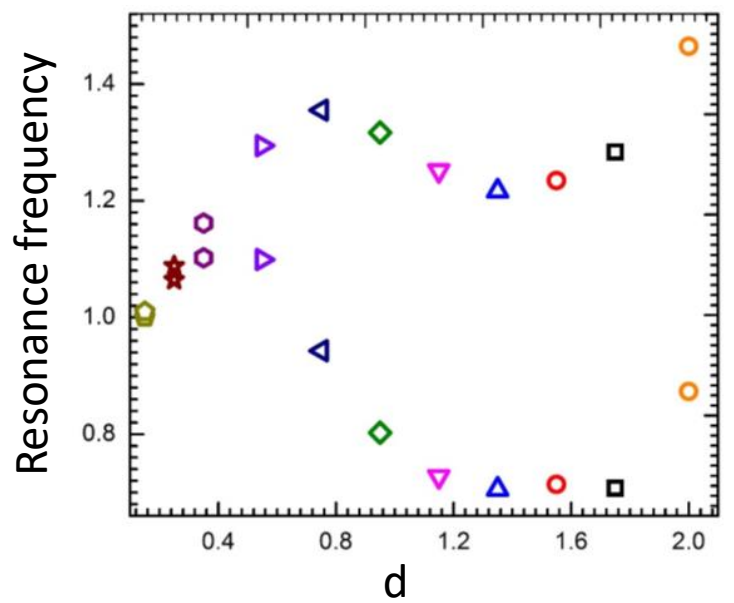

Figure 3: Resonant frequency with coupling beam thickness Changes in two resonance frequencies as the parameter ' $d$ ' is changed, as observed by finite element analysis

\section{READOUT ELECTRONICS}

Signal generated from these nano-mechanical resonators build of single layer graphene nano-structure is generally quite small and often buried in other parasitic or interference signals. In the present transduction scheme, the strain-dependent resistance produced in the graphene resonator due to the externally applied stress is measured. This piezoresistive readout method used here provides an efficient signal transduction mechanism from the mechanical motion to the electrical signal. Piezomechanical actuation is provided by a piezo-shaker. This piezo shaker is suitably attached to the device with necessary electrical insulation. A voltage signal $\left(\mathrm{V}_{\text {Ext }}\right)$ applied to the piezo shaker determines the input actuation

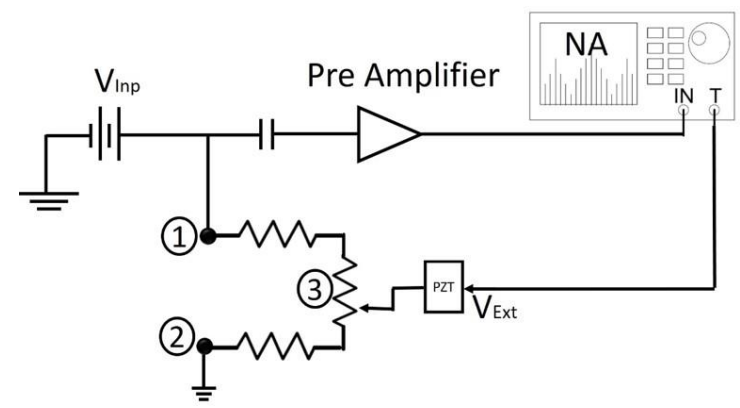

Figure 4: Measurement setup for the Piezoresistive Measurement from graphene Resonator, showing the schematic of the electric circuit of the mechanical resonator.

The mechanical motion of the graphene device leads to changes in its resistance. These changes are measured by applying a simple DC bias $\left(\mathrm{V}_{\text {Inp }}\right)$ to the device and measuring the resultant current. It is worth noting that the DC bias $\left(\mathrm{V}_{\text {Inp }}\right)$ also induces joule heating in the graphene resonator, hence varying $\mathrm{V}_{\text {Inp }}$ also provides a technique to vary the temperature of the resonator. By using a network analyzer, the resonance frequencies and the collective behaviour of the device was recorded. The measurement setup is shown in Figure 4. To ensure high quality signal recordings, all measurements were performed at low pressures of $\sim 4.0 \times 10^{-6}$ Torr. However, the device was operated at room temperature.

\section{RESULTS}

The frequency response, as recorded from the fabricated device is presented in Figure 5. Two resonance frequencies were observed as expected. The fabricated structure had three terminals. This enabled measurements of three different resistance values between combinations of any two of these terminals.

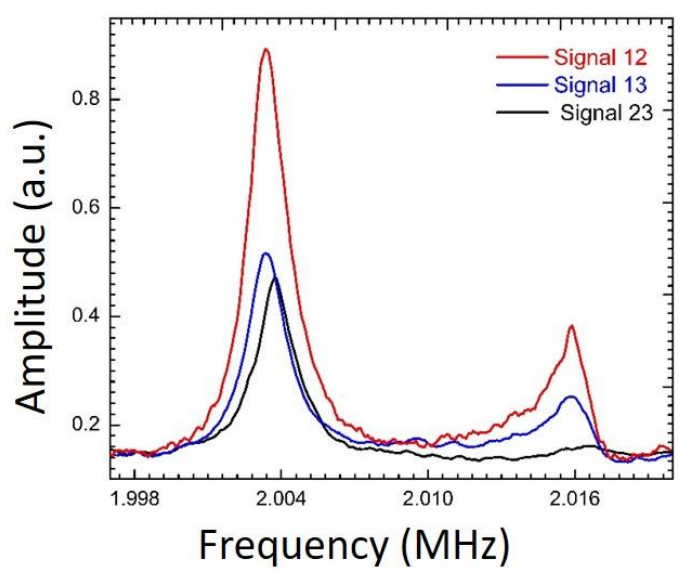

Figure 5: Experimental measurement of the frequency response clearly showing two resonance modes. Three waveforms as measured through the electrical potential difference between the three parts of the resonator, with all showing the two resonance peaks. Amplitude ratio of 1-2 and 2-3 is used to find eigenvectors.

Figure 5 also shows the frequency response, as measured between these three different terminals (1-2, $1-3$, and 2-3). The 1-3 and 2-3 measurements record the response of individual resonators. The ratio of the peak amplitudes can hence be used to measure the eigenvectors of the coupled system. A small change in the resonators is expected to lead to a significantly large change in this ration. At the same time, the resonance frequencies can actually be measured from the response of any one of these combinations. More importantly, the quality factor recorded from these devices is of the order of 1000 .

In addition to the response of individual resonators, the three-sided clamped structure also allows for a readout between the two resonators. The measurement between 
terminals ' $1-2$ ' adds the two responses of individual resonators, as it measures the entire resistance of the two resonators placed in series. This, hence provides a higher amplitude of detection, which will be very useful for frequency based sensors. The calculated mass sensitivity of this sensor is of the order of 10 Zeptogram. It is also worth noting that the structure has not been optimized and one can always change the collective behaviour response as well as amplitude by suitably designing the length, width and separation of the resonant beams and the coupling beam. This in turn will also lead to change of the sensitivity of the resonator array.

\section{CONCLUSIONS}

The paper reports the successful fabrication of a threesided clamped-clamped coupled graphene resonator measured at room temperature with a very high quality factor. Finite element simulations show the ability to systematically change the resonance frequencies with the aspect ratio of the resonators and coupling. As both resonators can be functionalized, this structure provides a two-in-one mechanically multiplexed sensing system. The response of any one resonator or the additive response of both resonators can be used as the sensor's response with different sensitivities. One can also use either the eigenvalue or the eigenvector for sensing. The results of the paper suggest that one can design a larger array of Graphene resonators thereby producing a coupled array of a large number of sensors. In addition, the presented geometry and the transduction mechanism provide simple electronic interface with a novel and highly sensitive sensing system. The future work will aim to build large arrays of such resonators.

\section{ACKNOWLEDGEMENT}

This work was supported by UK Engineering and Physical Sciences Council (EPSRC) via grants EP/N010159/1 and EP/M015173/1.

\section{REFERENCES}

[1] S. D. Senturia, Microsystem Design, Boston, MA, USA:Kluwer, 2001.

[2] K. L. Ekinci, M. L. Roukes, "Nanoelectro-mechanical systems", Rev. Sci. Instrum. Vol. 76, pp. 061101, 2005.

[3] J. S. Bunch, et al. "Electromechanical Resonators from Graphene Sheets", Science vol. 315, no. 5811, pp. 490493, 2007.

[4] A. Eichler, et al. "Nonlinear damping in mechanical resonators made from carbon nanotubes and graphene", Nature Nanotech., vol. 6, pp. 339-42, 2011.

[5] C. Chen, S. Rosenblatt, K. I. Bolotin, W. Kalb, P. Kim, I. Kymissis, H. L. Stormer, T. F. Heinz, and J. Hone, "Performance of monolayer graphene nanomechanical resonators with electrical readout", Nature Nanotech., vol. 4, no. 12, pp. 861-867, 2009.
[6] M. Kumar, H. Bhaskaran, "Ultrasensitive room temperature piezoresistive transduction in graphene based nanoelectromechanical systems" Nano Lett., p. 15 (4), pp. 2562-2567 (2015).

[7] A. K. Geim, and K. S. Novoselov, "The rise of graphene" Nat. Mater., Vol. 6, pp. 183-91, 2007.

[8] H. Hosseinzadegan, C. Todd, A. Lal, M. Pandey, M. Levendorf, J. Park. Graphene has ultra-high piezoresistive gauge factor. IEEE 26th International Conference on Micro Electro Mechanical Systems (MEMS), pp 611-614, 2012.

[9] C. Zhao et al., "A review on coupled MEMS resonators for sensing applications utilizing mode localization', Sensors and Actuators A, vol. 249, pp. 93-111, 2016.

[10] G. Tao, B. Choubey, “A Simple Technique to Readout and Characterize Coupled MEMS Resonators", IEEE/ASME J. Microelectromech. Syst., Vol. 25, Issue. 4, pp. 617-625, 2016.

[11] B. Choubey, E. J. Boyd, I. Armstrong, D. Uttamchandani, "Determination of the anisotropy of young's modulus using a coupled microcantilever array", J. Microelectromech. Syst., vol. 21, no. 5, pp. 1252-1260, Oct. 2012.

[12] B. E. DeMartini, J. F. Rhoads, S. W. Shaw, K. L. Turner, "A single input-single output mass sensor based on a coupled array of microresonators", Sens. Actuators A Phys., vol. 137, no. 1, pp. 147-156, 2007.

[13] G. Tao, H. Zhang, H. Chang, B. Choubey, "Inverse Eigenvalue Sensing in Coupled Micro/Nano System" IEEE/ASME J. Microelectromech. Syst., Vol. 27, Issue: 5, pp 886 - 895, Oct. 2018

[14] X. Song et al. "Stamp transferred suspended graphene mechanical resonators for radio frequency electrical readout", Nano Lett.,vol 12, pp. 198-202, 2011.

\section{CONTACT}

1. Prof. Bhaskar Choubey, tel: +49 - 271 -740 - 2466; bhaskar.choubey@uni-siegen.de

2. Dr. Madhav Kumar, madhav.kumar@cea.fr

3. Prof. Harish Bhaskaran, harish.bhaskaran@materials.ox.ac.uk 